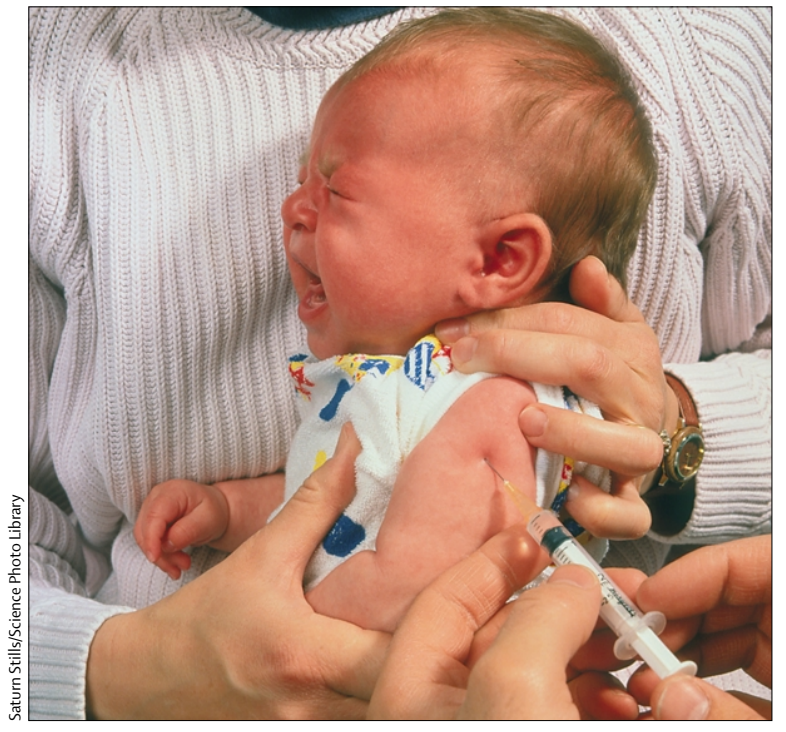

Are SCNA1 mutations waiting to be triggered by fever or stress?

mutations in SCN1A in 11 of 14 patients with purported vaccine encephalopathy. ${ }^{9}$

What is the reason for the temporal proximity of vaccination and onset of the epileptic encephalopathy? Is the SCN1A mutation a predisposing factor waiting to be triggered by fever or other stress? Probably so. In fact, as early as 2000, Nieto-Barrera and colleagues ${ }^{12}$ noted that more than $50 \%$ of patients with SMEI had their first seizure after DPT vaccination.

Should vaccination be continued in children diagnosed with SMEI, or for that matter with similar epileptic encephalopathies? This question awaits further study, but also needs careful consideration of each patient's particular clinical circumstances. Complications of naturally contracted infections are much higher than those associated with vaccination $(63 \%$ vs $7 \cdot 2 \%)$, and prophylactic antipyretic treatment in many cases can effectively prevent the common reactions to vaccination. ${ }^{13}$ In any case, a switchover to acellular pertussis is clearly warranted where this has not yet occurred.

The study by Berkovic and collaborators ${ }^{9}$ not only shows the novel association of SCN1A mutations with vaccine encephalopathy, but also helps to demystify the isolated association of vaccination and unexplained encephalopathy. This should help decrease unwarranted avoidance of vaccination and endangerment of children's and public health.

\section{Erick Sell, Berge A Minassian}

Division of Neurology, Department of Paediatrics, The Hospital for Sick Children, Toronto, ON, Canada bminass@sickkids.ca

We have no conflicts of interest.

1 American Academy of Pediatrics. 2003 red book: report of the Committee on Infectious Diseases, 26th edn. Elk Grove Village, IL, USA: American Academy of Pediatrics, 2003.

2 Piyasirisilp S, Hemachudha T. Neurological adverse events associated with vaccination. Curr Opin Neurol 2002; 15: 333-38.

3 Zhou W, Pool V, Iskander JK, et al. Surveillance for safety after immunization: Vaccine Adverse Event Reporting System (VAERS) - United States, 19912001. MMWR Surveill Summ 2003; 52: 1-24.

4 Bale LF. Neurologic complications of immunization. J Child Neurol 2004; 19: 405-12.

5 Geier D, Geier MR. Neurodevelopmental disorders following thiomersalcontaining childhood immunizations: a follow-up analysis. Int J Toxicol 2004 23:369-76.

6 Stehr-Green P, Tull P, Stellfeld M, Mortenson PB, Simpson D. Autism and thiomersal-containing vaccines: lack of consistent evidence for an association. AmJ Prev Med 2003; 25: 101-06.

7 Gale JL, Thapa PB, Wassilak SG, Bobo JK, Mendelman PM, Foy HM. Risk of serious acute neurological illness after immunization with diphtheriatetanus-pertussis vaccine: a population-based case control study. JAMA 1994; 271: 37-41

8 Geier DA, Geier MR. An evaluation of serious neurological disorders following immunization: a comparison of whole-cell pertussis and acellular pertussis vaccines. Brain Dev 2004; 26: 296-300.

9 Berkovic SF, Harkin L, McMahon JM, et al. De-novo mutations of the sodium channel gene $S C N 1 A$ in alleged vaccine encephalopathy: a retrospective study. Lancet Neurol 2006; 5: 488-92.

10 Dravet C. Les epilepsies graves de I'enfant. Vie Med 1978; 8: 543-48.

11 Claes L, Del-Favero J, Ceulemans B, Lagae L, Van Broeckhoven C, De Jonghe P. De novo mutations in the sodium-channel gene SCN1A cause severe myoclonic epilepsy of infancy. Am J Hum Genet 2001; 68: 1327-32.

12 Nieto-Barrera M, Lillo MM, Rodriguez-Collado C, Candau R, Correa A. Severe myoclonic epilepsy in childhood: epidemiological analytical study. Rev Neurol 2000; 30: 620-24.

13 Tanabe T, Awaya Y, Matsuishi T, et al. Survey of vaccination and viral infections for children with severe myoclonic epilepsy in infancy. No To Hattatsu 2004; 36: 318-23.

\title{
Antihypertensives for prevention of Alzheimer's disease
}

For about 25 years hypertension has been recognised as a long-term risk factor for Alzheimer's disease. The latent period between the manifestation of hypertension and the initial signs of mental deterioration is variable. The time lag first established in the Framingham study ${ }^{1}$ and subsequently confirmed by long-term studies with similar design and follow-up was of several decades, between hypertension in middle age (about age 50 years) and the first signs of cognitive dysfunction some 20 years later. With increasingly subtle diagnostic procedures, the temporal associations between hypertension and cognitive impairment seem to have become clearer. ${ }^{2}$ Developments 
of this kind unfortunately seem to escape the awareness of most clinicians in both general and specialist medicine.

Perhaps, the key problem is that most otherwise effective antihypertensive drugs do not provide a common generic guarantee of measurable protection against cognitive dysfunction by the lowering of blood pressure. Unfortunately, the large comparative prospective trials of antihypertensive drugs published over the past decade, which we recently reviewed, ${ }^{3}$ did not incorporate cognitive impairment or dementia as a trial endpoint. These trials have left us with few data, among which the dihydropyridine calcium-channel blocker nitrendipine, as used in the Systolic Hypertension in Europe trial, ${ }^{4}$ offered substantial protection against both vascular and degenerative dementias over up to 8 years of follow-up.

The recently reported Cache County Study ${ }^{5}$ is a realistic effort to offer some compensation for the missed opportunities of the large prospective trials. ${ }^{3}$ This multidisciplinary effort sought to establish evidence for specific cognitive protection by various antihypertensive treatments in a population of patients with hypertension age 65 years or older in a stable community. The ongoing study was started in 1995 (wave 1), including a personal interview and an adapted modified mini-mental state examination (3 MS). For those unable to participate in dialogue, a surrogate questionnaire (IQ CODE) was presented to a carer or family member. 3 years later, beginning in 1998, the procedure was repeated (wave 2). Those who then scored below predetermined cut-off points on the 3MS or the IQ CODE underwent a highly structured neuropsychological assessment. A preliminary diagnosis of dementia according to DSM-III-R criteria was followed by even more refined techniques including neuroimaging, and application of NINCDS-ADRDA (National Institute of Neurological and Communicative Disorders and Stroke and Alzheimer's Disease and Related Disorders Association) criteria to diagnose Alzheimer's disease. Participants without dementia from wave 1 were classified according to all antihypertensive drugs currently in use. Blood pressure was not taken in all participants, but merely in a fully examined subsample of patients. Covariables included routine factors, such as age, sex, education, cardiovascular illnesses, and the number of APOE alleles.

This vast, but in our view partly unfocused, exploration produced the following results. 3308 participants without dementia in wave 1 completed wave 2 to a degree sufficient to assess their cognitive status. Of these, 185 had dementia, which was diagnosed as Alzheimer's disease in 104 patients. The remaining 81 patients with dementia, presumably vascular or mixed dementias, were excluded from consideration for unspecified nosological reasons. In our opinion, the latter unfortunate decision could have wasted important data because degenerative and vascular dementia subtypes seem to be confluent in their substrates. ${ }^{6}$ Hopefully, the researchers will rerecruit this lost tribe.

The researchers reported an impressive gradation between the efficacies of the different antihypertensivedrug categories used in their study, in terms of preventing Alzheimer's disease (table). The big surprise here is the superior effect of potassium-sparing diuretics. It would be important to include the effect of these diuretics on the prevention of vascular dementia to see whether there are similar effects; this also applies to the apparent equivalence between dihydropyridine calcium-channel blockers and $\beta$-blockers. The rather poor effect of angiotensinconverting enzyme inhibitors came as no surprise to us, given the outcome of our earlier meta-analysis.?

The researchers rightly recommend further epidemiological and experimental studies with regard to possible explanations of the above results. As far as the dihydropyridine calcium-channel blockers are concerned, there is already a wealth of experimental studies explaining their potential protective mechanisms with regard to the prevention of both types of dementia. ${ }^{8}$ Whether or not lowering of blood pressure can prevent Alzheimer's disease and to what extent some antihypertensive drugs can provide specific protection against neurodegenerative dementia, above and beyond blood-pressure lowering, is an issue with far-reaching implications for public health. Because of the worldwide demographic transition from high to low rates of birth and death, dementia is fast becoming one of the principal causes of major disability and

\begin{tabular}{|ll|}
\hline & Adjusted HR $(95 \% \mathrm{CI})$ \\
\hline Potassium-sparing diuretics & $0.26(0.08-0.64)$ \\
Dihydropyridine CCBs & $0.53(0.16-1 \cdot 34)$ \\
$\beta$-blockers & $0.53(0.22-1 \cdot 09)$ \\
Diuretics & $0.53(0.22-1 \cdot 09)$ \\
ACE inhibitors & $1.13(0.60-1 \cdot 98)$ \\
HR=hazard ratio; $C \mathrm{CB}=$ calcium-channel blocker; ACE=angiotensin converting enzyme. \\
\hline Table: Association of Alzheimer's disease with use of antihypertensive \\
drugs
\end{tabular}


mortality. ${ }^{6,9}$ Medical treatment of established dementia has only marginal benefit and is not cost effective. Prevention is the only way to turn the tide. Clinical trials must be begun to specifically address the question of whether drug class matters in the prevention of Alzheimer's disease by blood-pressure lowering. ${ }^{9}$ Observational studies, such as the Cache County Study from Utah, ${ }^{5}$ even when properly analysed, are subject to biases originating from reverse causality, attrition, and self-selection of participants. Such studies are only hypothesis-generating and will never provide a definite answer.

Willem H Birkenhäger, Jan A Staessen

Erasmus University Rotterdam, Netherlands (W H Birkenhäger) and Studies Coordinating Centre, Division of Hypertension and Cardiovascular Rehabilitation, Department of Cardiovascular Diseases, University of Leuven, Belgium (J A Staessen) w.birkenhager@chello.nl
We have no conflicts of interest.

The authors gratefully acknowledge the assistance of Sandra Covens and Katrien Staessen.

1 Elias ME, Wolf PA, D'Agostino RB, et al. Untreated blood pressure level is inversely related to cognitive functioning: the Framingham Study. Am J Epidemiol 1993; 138: 353-64.

2 Elias PK, Elias MF, Robbins MA, et al. Blood pressure-related cognitive decline: does age make a difference? Hypertension 2004; 44: 631-36.

3 Staessen JA, LiY, Thijs L, Wang JG. Blood pressure reduction and cardiovascular prevention: an update including the 2003-2004 secondary prevention trials. Hypertens Res 2005; 28: 385-407.

4 Forette F, Seux ML Staessen JA, et al. The prevention of dementia with antihypertensive treatment: new evidence from the Systolic Hypertension in Europe (Syst-Eur) Trial. Arch Intern Med 2002; 162: 2046-52.

5 Khachaturian AS, Zandi PP, Lyketsos CG, et al. Antihypertensive medication use and incident Alzheimer disease. Arch Neurol 2006; 63: E1-E7.

6 Casserly I, Topol E. Convergence of atherosclerosis and Alzheimer's disease: inflammation, cholesterol, and misfolded proteins. Lancet 2004; 363: 1139-46.

7 Wang JG, Staessen JA, Birkenhäger WH. Antihypertensive treatment and prevention of stroke and dementia. Semin Cerebrovasc Dis Stroke 2003; 3: $155-60$.

8 Birkenhäger WH, Staessen JA. Cognitive function in essential hypertension. Prog Cardiovasc Dis 2006 (in press).

9 Staessen JA, Birkenhäger WH. Cognitive impairment and blood pressure; quo usque tandem abutere patientia nostra? Hypertension 2004; 44: 612-13.

\section{Cholinesterase inhibitors in late-stage Alzheimer's disease}

The Swedish Nursing Home Study ${ }^{1}$ compared donepezil with placebo for the treatment of severe Alzheimer's disease in patients living in assisted-care facilities; this study offers an opportunity to think about the use of cholinesterase inhibitors in late stages of this disorder. Winblad and colleagues used standard outcomes to assess the efficacy of donepezil: the severe impairment battery $(\mathrm{SIB})$, the cognitive measure most appropriate for this stage of Alzheimer's disease; an activity of daily living measure modified for severe Alzheimer's disease (ADCS$\mathrm{ADL}$-severe); the neuropsychiatric inventory (the most

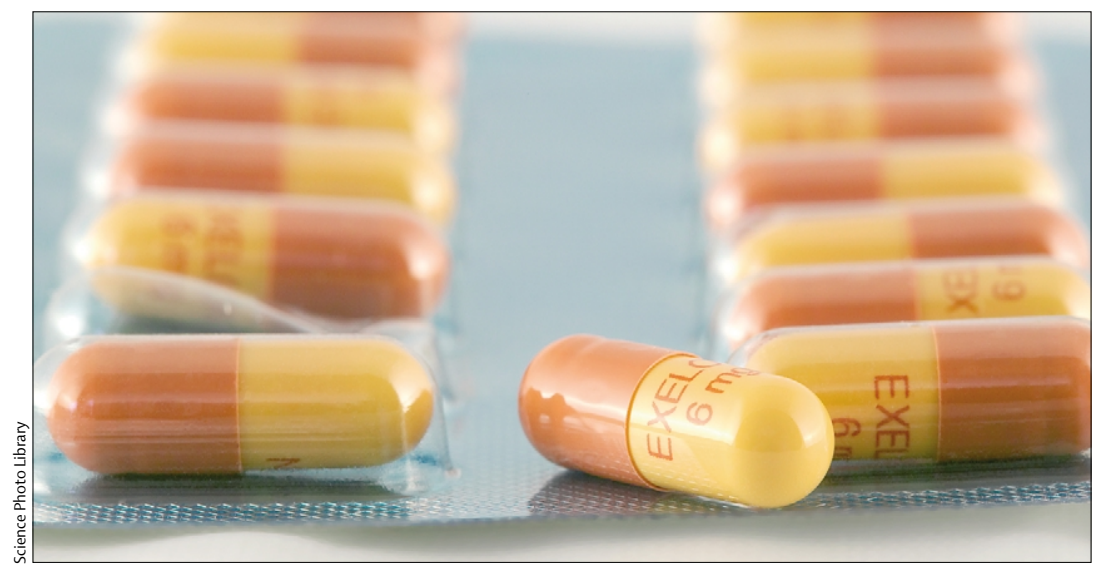

Cholinesterase inhibition in Alzheimer's disease may need to be tailored to individual patients widely used behavioural measure); the mini-mental state examination (MMSE), which is not as sensitive to change in this disease stage; and a clinical global impression of improvement. The primary analysis on a modified intention-to-treat population showed significant differences in favour of donepezil in SIB, ADCS-ADLsevere, and the MMSE but not for the neuropsychiatiric inventory or clinical global impression of improvement. Adverse events were equivalent except for diarrhoea and hallucinations, which were more than twice as common in patients taking donepezil than in those taking placebo. The researchers had predetermined a clinically significant treatment difference for the two primary outcomes (SIB and ADCS-ADL), which was not quite reached at 6 months, as highlighted in the accompanying editorial by David Hogan. ${ }^{2}$

Another study comparing donepezil with placebo in community-dwelling patients with severe Alzheimer's disease showed benefits for all outcomes, including the total neuropsychiatric inventory score at 6 months. ${ }^{3}$ The inability to detect improvement of behaviour in the Swedish Nursing Home Study may be caused by the sensitivity of the neuropsychiatric inventory in a nursinghome setting, the fact that non-family members are asked about their perception of the patients' moods 